\title{
Influence of Atomic Scale Compositional Gradients on Colossal Ionic Conductivity in Highly Strained YSZ/STO Heterostructures
}

\author{
David W. McComb ${ }^{1}$, Frank J Scheltens ${ }^{1}$, Jacobo Santamaria ${ }^{2}$, Carlos Leon ${ }^{2}$, Alberto Rivera ${ }^{2}$ \\ 1. Department of Materials Science and Engineering, The Ohio State University \\ College Road, Columbus OH 43210, USA \\ 2. Grupo de Fisica de Materiales Complejos, Universidad Complutense, 28040 Madrid, Spain
}

There have been numerous reports in the literature of significantly enhanced ionic conductivity in multilayer heterostructures formed from ionic conductors and insulators. [1-3] There have also been a number of reports suggesting that these enhancements are due to electronic rather than ionic conductivity. [4,5] Many of these reports have focused on heterostructures between SrTiO3 (STO) and yttria-stabilised zirconia (YSZ). [3,5]

It would be highly informative to understand how the lattice mismatch ( $>7 \%)$ between STO and YSZ is accommodated. It has been suggested that a highly disordered region is present at each interface in a trilayer structure, which results in high local concentration of oxygen vacancies. Electron energy-loss spectroscopy (EELS) in the scanning transmission electron microscope (STEM) has been used to address this question but close proximity of the atomic numbers (Sr:38, Y:39, Zr:40) make overlap of ionization edges problematic. [5,6] In this contribution we will report on the new insights obtained using high resolution energy-dispersive X-ray (EDX) analysis in the STEM.

STEM, EELS and EDX measurements were performed on an FEI Titan 60-300 (S)TEM operated at $300 \mathrm{kV}$ using a high brightness Schottky field emission electron source, an aberration corrector, Super-X quadrant EDX system and Quantum EELS spectrometer. Some data were also obtained on an 80-200kV Titan ChemiSTEM instrument. The YSZ/STO heterostructures were grown in a high oxygen pressure (3 mbar) pure O rf sputtering system and prepared in cross section using FIB methods and low energy ion milling. The samples were tilted in the microscope to either the (100) or (110) cubic zone axis of STO.

Fig. 1 shows a HAADF STEM image of a nominal $1 \mathrm{~nm}$ thick YSZ layer that is coherent with the STO structure above and below. EDX analysis reveals that the Y/Zr ratio varies systematically from bottom to top in this layer, and the atomic correlation with the sub-lattices in the STO structure suggest that the YSZ layer does not exhibit a fluorite structure.

We conclude that highly strained YSZ layers epitaxially grown on STO exhibit systematic compositional fluctuations on the atomic scale that may be associated with the formation of a metastable non-fluorite structure. 


\section{References:}

[1] Kosacki, I., Rouleau, C. M., Becher, P. F., Bentley, J., \& Lowndes, D. H. (2005). Solid State Ionics, 176(13-14), 1319-1326.

[2] Sata, N., Jin-Phillipp, N. Y., Eberl, K., \& Maier, J. (2002). Solid State Ionics, 154, 497-502.

[3] Garcia-Barriocanal, J., Rivera-Calzada, A., Varela, M., Sefrioui, Z., Iborra, E., Leon, C., Pennycook, S.J. \& Santamaria, J. (2008). Science, vol. 321, no. 5889, pp. 676-680.

[4] Guo, X. (2011). Scripta Materialia, 65(2), 96-101.

[5] Cavallaro, A., Burriel, M., Roqueta, J., Apostolidis, A., Bernardi, A., Tarancon, A., Srinivasan, R., Cook, S.N., Fraser, H.L., Kilner, J.A., McComb, D.W. \& Santiso, J. (2010). Solid State Ionics, vol. 181, no. $13-14$, pp. 592-601

[6] Pennycook, T.J., Oxley, M.P., Garcia-Barriocanal, J., Bruno, F.Y., Leon, C., Santamaria, J., Pantelides, S.T., Varela, M. \& Pennycook, S.J. (2011). European Physical Journal-Applied Physics, vol. 54, no. 3, pp. 33507

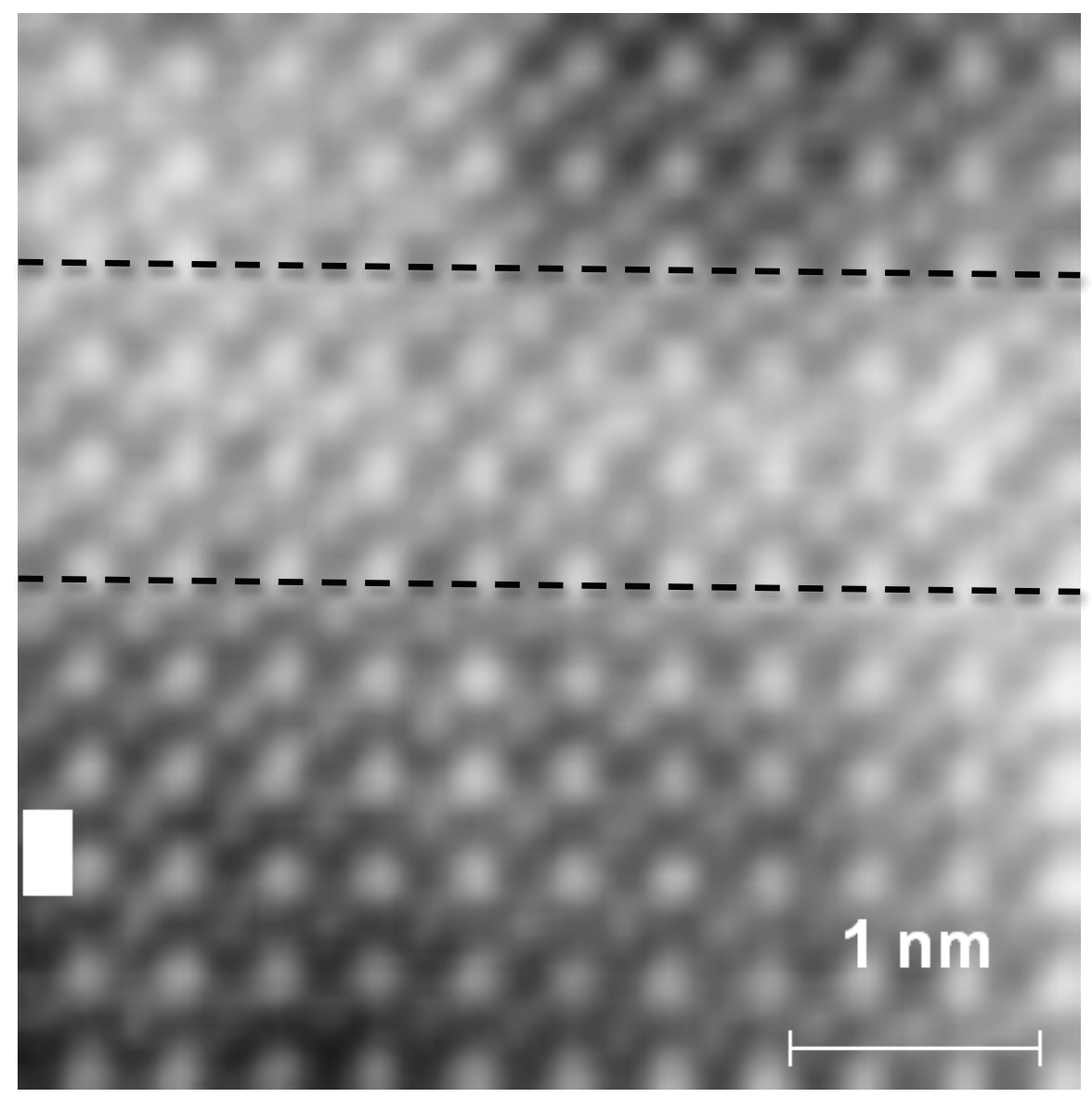

Figure 1. HAADF STEM image of a coherent YSZ layer (dotted lines) with STO above and below. 